\title{
Direct decay-energy measurement as a route to the neutrino mass
}

\author{
J. Karthein · D. Atanasov* • K. Blaum • \\ S. Eliseev • P. Filianin • D. Lunney • \\ V. Manea ${ }^{\text {* }}$. M. Mougeot ${ }^{\dagger}$. D. Neidherr • \\ Y. Novikov • L. Schweikhard • A. Welker • \\ F. Wienholtz • K. Zuber
}

Received: January 29, 2019 / Accepted: May 14, 2019

\begin{abstract}
A high-precision measurement of the ${ }^{131} \mathrm{Cs} \rightarrow{ }^{131} \mathrm{Xe}$ ground-to-groundstate electron-capture $Q_{\mathrm{EC}}$-value was performed using the ISOLTRAP mass spectrometer at ISOLDE/CERN. The novel PI-ICR technique allowed to reach a relative mass precision $\delta m / m$ of $1.4 \cdot 10^{-9}$. A mass resolving power $m / \Delta m$ exceeding $1 \cdot 10^{7}$ was obtained in only $1 \mathrm{~s}$ trapping time. Allowed electron-capture transitions with sub-keV or lower decay energies are of high interest for the direct determination of the $\nu_{e}$ mass. The new measurement improves the uncertainty on the ground-to-ground-state $Q_{\mathrm{EC}}$-value by a factor 25 precluding the ${ }^{131} \mathrm{Cs} \rightarrow{ }^{131} \mathrm{Xe}$ pair as a feasible candidate for the direct determination of the $\nu_{e}$ mass.
\end{abstract}

Keywords PI-ICR · $\beta$-decay · neutrino mass · high-precision mass spectrometry

* Current address: KU Leuven, Instituut voor Kern- \& Stralingsfysica, 3001 Leuven, Belgium

$\dagger$ Current address: Max-Planck-Institut für Kernphysik, 69117 Heidelberg, Germany

This article contains data from the Ph.D thesis work of Jonas Karthein, enrolled at Heidelberg University, Germany. Corresponding author: jonas.karthein@cern.ch

J. Karthein · V. Manea · A. Welker · F. Wienholtz

CERN, Route de Meyrin, 1211 Genève, Switzerland

J. Karthein · K. Blaum · S. Eliseev · P. Filianin

Max-Planck-Institut für Kernphysik, 69117 Heidelberg, Germany

D. Atanasov · A. Welker · K. Zuber

Technische Universität Dresden, 01062 Dresden, Germany

D. Lunney · M. Mougeot

CSNSM-IN2P3-CNRS, Université Paris-Sud, 91400 Orsay, France

D. Neidherr

GSI Helmholtzzentrum für Schwerionenforschung, 64291 Darmstadt, Germany

Y. Novikov

Department of Physics, St Petersburg State University, St Petersburg 198504, Russia

Y. Novikov

Petersburg Nuclear Physics Institute, 188300 St Petersburg, Russia

L. Schweikhard · F. Wienholtz

Physikalisches Institut, Universität Greifswald, 17489 Greifswald, Germany 


\section{Introduction}

The determination of the neutrino rest mass is of broad interest not only in nuclear physics but also in the fields of particle and astrophysics. On the most fundamental level, the existence of a non-zero neutrino mass is not explained by the standard model. However, abundant experimental evidence by the observation of neutrino oscillations has been found in the last decades, which requires a neutrino mass and mixing. Hence, a detailed study of different neutrino properties and interactions evolved as a powerful tool in the search for the fundamental theory beyond the standard model. [1, 2] A very feasible approach for the determination of the electron-neutrino mass lies in the investigation of electron-capture (EC) reactions with energies of a few $\mathrm{keV}$ or lower. Here, the only particle emitted is the neutrino itself. Therefore, the smaller the decay energy of these transitions, the higher the sensitivity to the neutrino rest mass. Such transitions are found in allowed ECtransitions to excited nuclear states in the daughter nucleus.

Electron and nuclear excitation energies are typically known to sub-keV precision. Unfortunately, the ground state masses of the decay pairs are, in most cases, known with uncertainties well above $1 \mathrm{keV}$ and thus constitute the main contribution to the uncertainty of decay energies. Presently, only Penning-trap mass spectrometry (PTMS) is capable of providing mass measurements with sub-keV uncertainties. In recent years, a combination of PTMS and cryogenic microcalorimetry (MMC) 3 has proven to be a very successful combination for investigating the $\beta^{-}$-decay in ${ }^{187}$ Re and the electron capture in ${ }^{163}$ Ho [4. Several other transitions have been subsequently suggested as possible candidates for neutrino physics research - the electron-capture of ${ }^{131} \mathrm{Cs}$ to the $E^{*}=364.490(4) \mathrm{keV}$ [ excited state in ${ }^{131} \mathrm{Xe}$ being one example.

\section{Experiment and analysis}

The measurement was performed with the high-precision Penning-trap mass spectrometer ISOLTRAP [6, 7, 8] located at CERN's radioactive ion beam facility ISOLDE 9]. There, isotopes are produced in nuclear reactions in a thick target, induced by a $1.4 \mathrm{GeV}$ proton beam. In the present case a uranium-carbide target was used. After surface ionization, the beam was accelerated to $50 \mathrm{keV}$, magnetically separated for the ion of interest in ISOLDE's HRS separator and transported to the ISOLTRAP setup.

The ISOLTRAP apparatus, depicted in Fig. 1, consists of a sequence of four ion traps. The continuous ${ }^{131} \mathrm{Cs}^{+}$beam from ISOLDE, as well as the ${ }^{133} \mathrm{Cs}^{+}$beam from ISOLTRAP's offline alkali ion source in the case of reference mass, is first accumulated in a radio-frequency quadrupole (RFQ) trap [10, where it is cooled and bunched for $10 \mathrm{~ms}$ using ultra-pure helium gas. Isobaric separation is subsequently performed using ISOLTRAP's multi-reflection time-of-flight (MR-ToF) device [11, in which trapped ions are reflected back and forth in order to extend their flight path to $\sim 1 \mathrm{~km}(\sim 28 \mathrm{~ms})$. Not only has this device shown numerous times its suitability for the measurement of short-lived isotopes produced in min- 


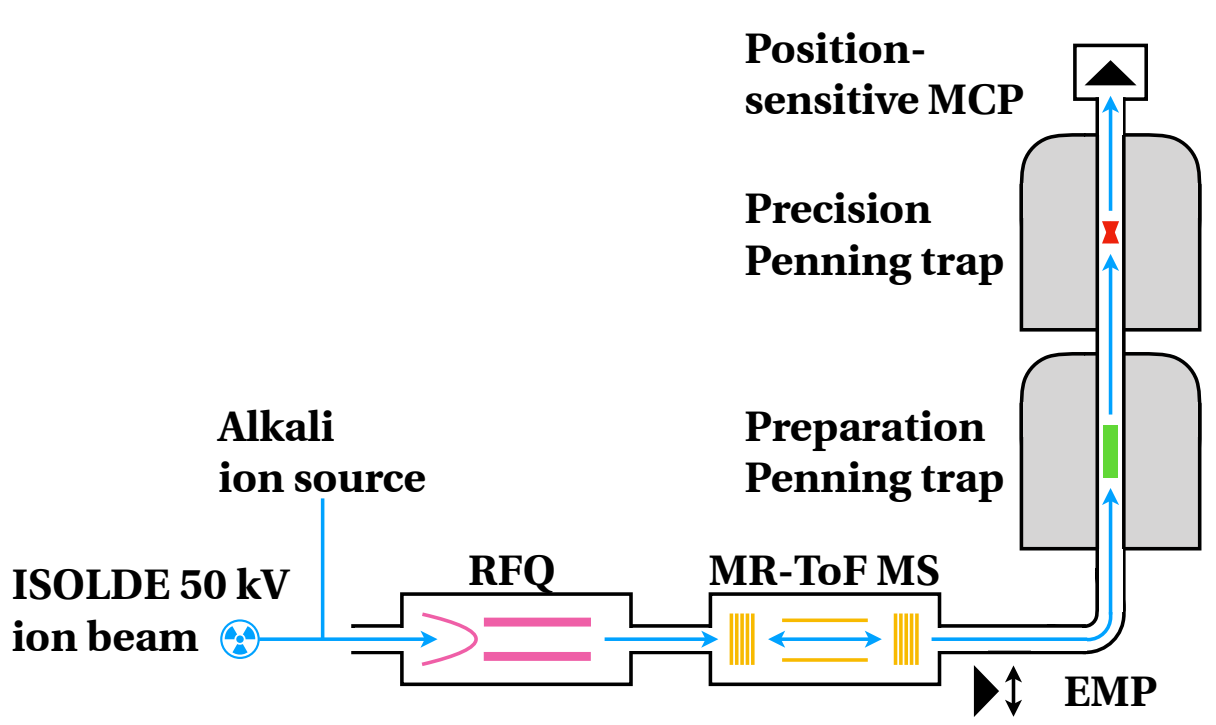

Fig. 1 Schematic overview of the ISOLTRAP mass spectrometer. Radioactive ion beams provided by ISOLDE or an offline alkali ion source at an energy of $50 \mathrm{keV}$ are delivered. Inside the ISOLTRAP apparatus the beam is processed by a sequence of traps: a radio-frequency quadrupole (RFQ) cooler and buncher (pink), a multi-reflection time-of-flight (MR-ToF) mass separator/spectrometer (yellow), a preparation Penning trap (green) and a precision Penning trap (red). Furthermore, an electron multiplier (EMP) particle detector for ToF detection and a position-sensitive multi-channel plate (MCP) particle detector for position and ToF detection are shown. For further details, see text.

utes quantities [12, 13] but it has also proved itself to be a perfectly suitable tool for mass purification [14. More specifically, in this experiment a mass resolving power $R=m / \Delta m=t /(2 \cdot \Delta t)$ (where $t$ is the mean of the time-of-flight distribution and $\Delta t$ its full width at half maximum) in excess of $1.1 \cdot 10^{5}$ was achieved. The purified beam is then transported to the helium buffer-gas-filled preparation Penning trap for further cooling and purification following the well-established mass-selective centering technique [15. Ultimately, the ions arrive in the precision Penning trap where high-precision mass determination is accomplished by measuring the ion's cyclotron frequency $\nu_{c}$

$$
\nu_{c}=\frac{1}{2 \pi} \cdot \frac{q_{i}}{m_{i}} \cdot B
$$

with the charge-to-mass ratio $q_{i} / m_{i}$ and the magnetic field strength $B$. All detection techniques currently available at the ISOLTRAP setup - namely the single pulse time-of-flight ion-cyclotron-resonance (ToF-ICR) mass spectrometry (MS) [16, the two-pulse Ramsey-type ToF-ICR MS [17] and the recently developed phase-imaging ion-cyclotron-resonance (PI-ICR) MS [18, 19] - were all used in the presented experiment.

In both ToF-ICR techniques an excitation frequency is scanned, i.e. the excitation frequency is varied from one experimental cycle to the next, and the ion's time of flight (ToF) to a multi-channel plate detector after ejection from the trap is measured. This ToF has a minimum at the cyclotron frequency. A typical Ramsey-type ToF-ICR scan for ${ }^{131} \mathrm{Cs}^{+}$is shown in Fig. 2 for an excitation time of $100 \mathrm{~ms}$ per 


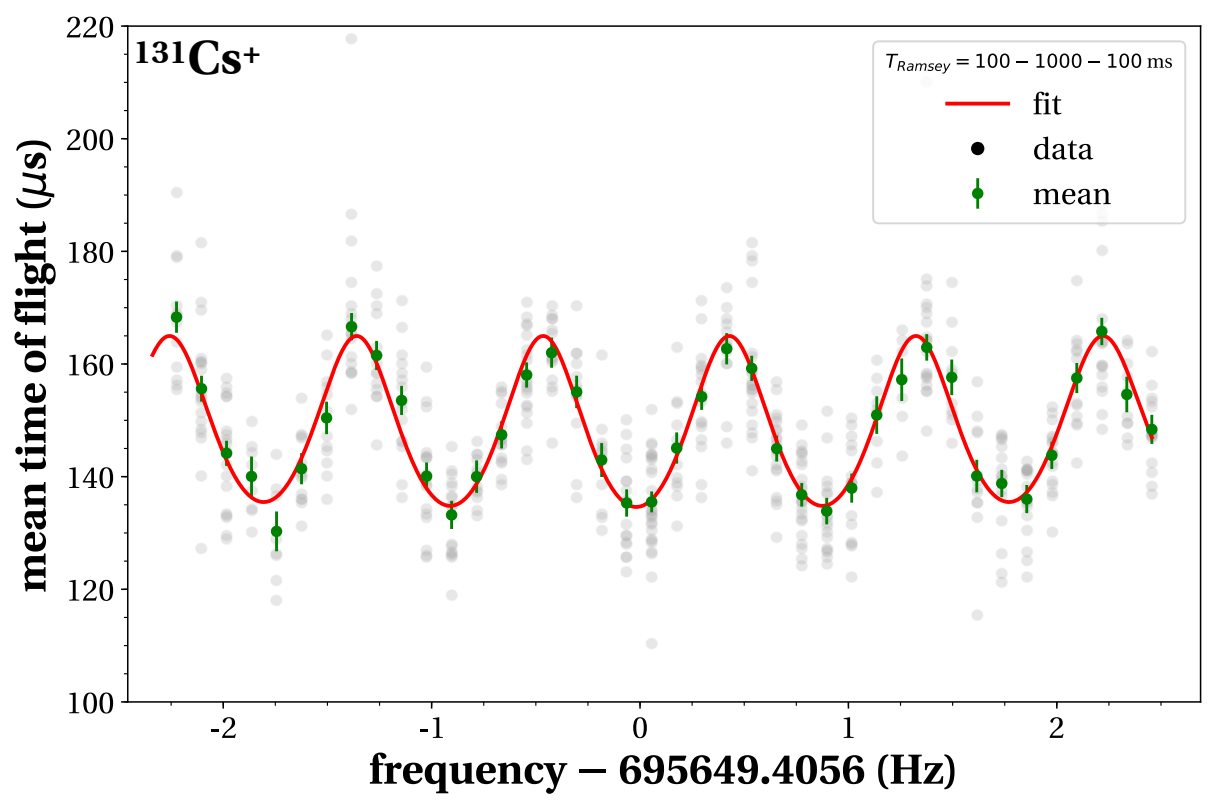

Fig. 2 Typical Ramsey-type ToF-ICR spectrum of ${ }^{131} \mathrm{Cs}^{+}$with an excitation time of $100 \mathrm{~ms}$ per pulse and $1000 \mathrm{~ms}$ waiting time. Individual, repeated ToF measurements are shown in black without any analysis cuts, thus demonstrating the purity of the beam injected inside the precision Penning trap. The mean of the unbinned ToF distribution per scan step with its standard deviation as error bar and the fitted theoretical line shape are represented in green and red respectively [16]. For further details, see text.

pulse and a "waiting time" of $1000 \mathrm{~ms}$ between the pulses. There, the individual, repeated ToF measurements per scan step is shown in black. The green data points represent the mean of the unbinned ToF distribution per scan step with its standard deviation as error bar. The red line represents a least squares fit of the theoretical line shape to the mean ToF distributions [16].

In addition to the well-established ToF-ICR techniques, the new non-scanning approach to PTMS, namely PI-ICR, has been applied. This method allows the determination of radial ion frequencies by determining the full phase $\phi_{\text {tot }}=2 \pi n+\phi$ in a given accumulation time $t_{\mathrm{acc}}$, consisting of an integer number $n \in \mathbb{N}_{0}$ of full turns plus an additional phase $\phi$ which is measured. The radial frequency then results as $\nu_{i}=(2 \pi n+\phi) /\left(2 \pi t_{\text {acc }}\right)$. Since the cyclotron frequency in a Penning trap $\nu_{c}=\nu_{+}+\nu_{-}$is equal to the sum of its radial eigenfrequencies $\nu_{+} /_{-}$, the technique is perfectly suited for PTMS allowing a frequency determination at the same or better precision as ToF-ICR techniques with $\sim 25$ times shorter measurement time [19].

A typical PI-ICR detector image for ${ }^{131} \mathrm{Cs}^{+}$is shown in Fig. 2. The dots represent repeated position projections (so called spots) from the Penning trap to a position-sensitive detector. In this case, the frequency determination was performed according to the pattern 1/2 (in Fig. 3 referred to as P1/P2) measurement scheme described in 19 which allows for a direct determination of $\nu_{c}$. The achieved 


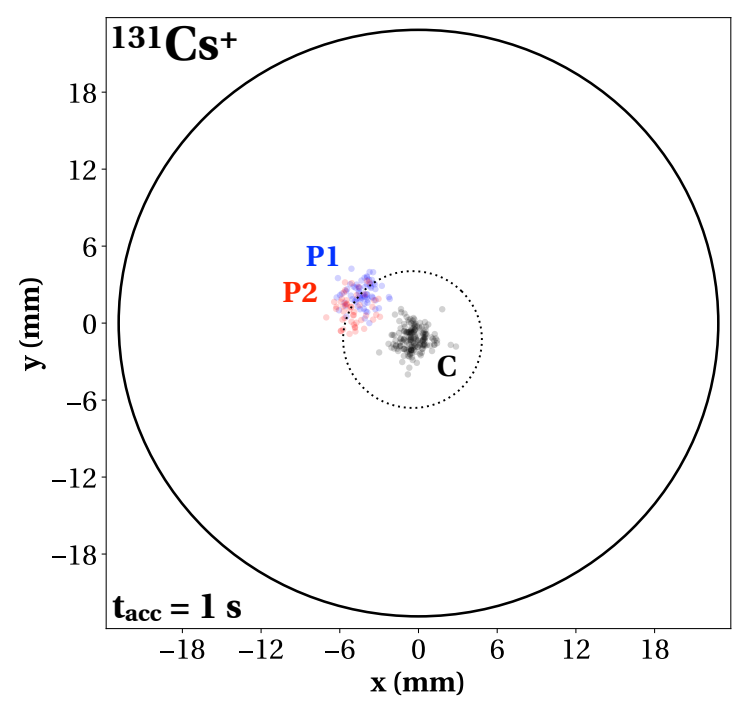

Fig. 3 Typical PI-ICR detector image for ${ }^{131} \mathrm{Cs}^{+}$with a center spot $\mathbf{C}$ and two overlapping spots P1/P2 for $t_{\mathrm{acc}}=1 \mathrm{~s}$, resolving power $R=\phi_{\mathrm{tot}} /(2 \cdot \Delta \phi)=1 \cdot 10^{7}$. For further details, see text and Ref. [19].

resolving power $R$ in case of Fig. 3 was $R=\phi_{\text {tot }} /(2 \cdot \Delta \phi)=1 \cdot 10^{7}$ with the total accumulated phase $\phi_{\text {tot }}$ after $t_{\text {acc }}=1 \mathrm{~s}$ and the spot's FWHM in terms of angle $\Delta \phi$. The analysis was performed with a custom-designed analysis software (for details see Ref. [20]) based on Python and ROOT [21. The analysis was independently performed with a LabView analysis software developped by the SHIPTRAP collaboration [19] and agrees within uncertainties.

The determination of the cyclotron frequency ratio $r=\nu_{\mathrm{c}, \text { ioi }} / \nu_{\mathrm{c}, \text { ref }}$ between all measured cyclotron frequency values $\nu_{c}$,ioi of the ion of interest (in this case ${ }^{131} \mathrm{Cs}^{+}$) of all three measurement methods and the reference ion values $\nu_{c \text {,ref }}$ (in this case ${ }^{133} \mathrm{Cs}^{+}$) is performed by simultaneously fitting a polynomial function $p(t)$ to both data sets [22]:

$$
\begin{aligned}
& \nu_{\mathrm{c}, \text { ioi }}=p(t) \\
& \nu_{\mathrm{c}, \text { ref }}=r \cdot \nu_{\mathrm{c}, \text { ioi }}=r \cdot p(t) .
\end{aligned}
$$

The polynomial fit function describes the temporal evolution of the cyclotron frequencies while the proportionality between the two fits is exactly the cyclotron frequency ratio $r$. The ground-to-ground state $Q_{\mathrm{EC}}$-value can be directly expressed following the relation:

$$
Q_{\mathrm{EC}}=(r-1) \cdot\left(m_{\mathrm{ref}, \mathrm{lit}}-m_{e}\right),
$$

where $m_{\text {ref,lit }}$ is the literature mass of the reference ion (here taken from AME16 [23]) and $m_{e}$ [24] is the electron mass. Figure 4 shows all individual cyclotron frequency measurements of ${ }^{131} \mathrm{Cs}^{+}$and ${ }^{133} \mathrm{Cs}^{+}$over time. In addition, the polynomial fits are shown. As one can see, all PTMS detection methods used in this publication are in good agreement. Moreover, the weighted mean of all individual 


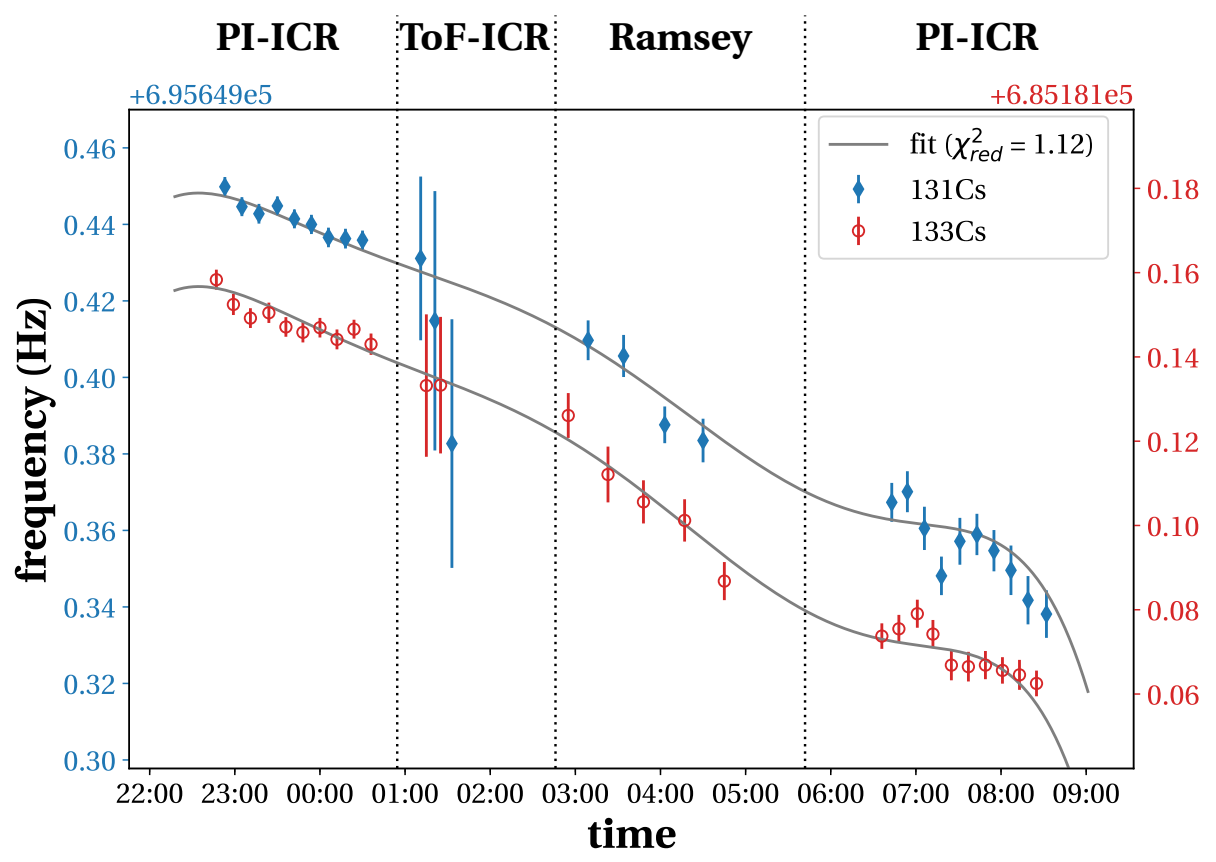

Fig. 4 Simultaneous polynomial fits of the four data sets as well as of all cyclotron frequency data for ${ }^{131} \mathrm{Cs}^{+}$and ${ }^{133} \mathrm{Cs}^{+}$. For further details, see text.

cyclotron frequency ratios for neighboring, alternating frequency measurements of ${ }^{131} \mathrm{Cs}^{+}$and ${ }^{133} \mathrm{Cs}^{+}$was calculated [25] and agrees with the polynomial method described above. The final frequency ratio yields $r_{\text {final }}=0.9849517704(14)$. The uncertainty of the combination of all PI-ICR data is $\delta \nu_{c} / \nu_{c}=1.4 \cdot 10^{-9}$.

In addition to the statistical uncertainty derived from the fit, a careful analysis of the systematic uncertainties which are not covered by the polynomial fit was performed. These include considering fit parameter correlations, where off-axis elements in the correlation matrix were negligibly small. The fluctuation of the individual frequencies after applying different fit cuts was systematically studied. They were found to be well within the statistical uncertainty on the individual frequency, proofing the purity of the beam. Since the ion rate was purposely kept below one ion per measurement cycle, a z-class analysis, i.e. reducing the number of detected ions per cycle and therefore in the trap itself, did not have to be performed. The data was corrected for ISOLTRAP's mass-dependent shift (relative shift: $7 \cdot 10^{-10}$ ) due to the difference in mass between the ion of interest and the reference ion as described in Ref. [25, 26]. The residual systematic uncertainty of ISOLTRAP [25] was not taken into account due to the fact that both the ion of interest and the reference were prepared, injected and measured in identical conditions, hence probing the same volume of the precision trap.

Table 1 presents the obtained ground-to-ground-state decay energy $Q_{\mathrm{EC}}$ as well as the allowed $\left(Q_{\mathrm{EC}}-E^{*}\right)$-value of interest to the $E^{*}=364.490(4) \mathrm{keV}[5]$ state in ${ }^{131} \mathrm{Xe}$ with their associated uncertainties. The decay-energy of the allowed EC- 
Table 1 Comparison of the measured mass excess, the measured released energy $Q_{\mathrm{EC}}$ of the electron-capture-pair ground-to-ground-state decay of ${ }^{131} \mathrm{Cs} \rightarrow{ }^{131} \mathrm{Xe}$, the released energy $\left(Q_{\mathrm{EC}}-E^{*}\right)$ for this electron-capture-pair in terms of ground-to-excited-state decay of ${ }^{131} \mathrm{Cs} \rightarrow{ }^{131} \mathrm{Xe}^{*}$, the latter one corrected for the binding energy $B$ of captured $L$ - and $M$-shell electrons $\left(Q_{\mathrm{EC}}-E^{*}-B\right)$ and the final uncertainty compared to literature [5, 23. For further details, see text.

\begin{tabular}{ccccccc}
\hline$(\mathrm{keV})$ & $M E$ & $Q_{\mathrm{EC}}$ & $Q_{\mathrm{EC}}-E^{*}$ & $Q_{\mathrm{EC}}-E^{*}-B_{L}$ & $Q_{\mathrm{EC}}-E^{*}-B_{M}$ & unc. \\
\hline Literature & -88059 & 355 & -10 & -15 & -11 & 5 \\
ISOLTRAP & -88055.56 & 358.00 & -6.49 & -11.95 & -7.64 & 0.17 \\
\hline
\end{tabular}

transition has to be corrected for the binding energy $B$ of captured electrons $\left(Q_{\mathrm{EC}}-E^{*}-B\right)$ to the $L$-shell-electron $\left(B\left(L-e^{-}\right)=5.453 \mathrm{keV}[27,28]\right)$ and to the $M$-shell-electron $\left(B\left(M-e^{-}\right)=1.1487 \mathrm{keV}\right.$ [27, 28]). It is worth mentioning, that the ${ }^{131} \mathrm{Xe}$ literature mass is dominated by a high-precision measurement from SHIPTRAP using the PI-ICR technique [29].

With the refined uncertainty, the ground-to-excited-state value $\left(Q_{\mathrm{EC}}-E^{*}\right)=$ $-6.49(17) \mathrm{keV}$ appears undoubtedly negative. This translates to the excited state ${ }^{131} \mathrm{Xe}^{*}$ being higher in energy than the parent ground state in ${ }^{131} \mathrm{Cs}$, thus prohibiting this ${ }^{131} \mathrm{Cs} \rightarrow{ }^{131} \mathrm{Xe}^{*}$ transition and excluding it as a suitable candidate for the determination of the electron-neutrino mass.

\section{Conclusion}

High-precision mass measurements of the ${ }^{131}$ Cs using established time-of-flight ion-cyclotron-resonance (ToF-ICR) mass spectrometry (MS) as well as the recently developed phase-imaging ion-cyclotron-resonance (PI-ICR) detection technique was performed with ISOLTRAP/CERN. We were able to demonstrate the successful implementation of PI-ICR at ISOLTRAP with a high resolving power of $1 \cdot 10^{7}$ for $1 \mathrm{~s}$ single-measurement time, a statistical uncertainty of only $1.4 \cdot 10^{-9}$ in $\sim 4$ hrs of beam time and a very good agreement with our well-established ToFICR measurement techniques (see Fig. 4). The obtained $Q_{\mathrm{EC}-\text { value agrees with }}$ the value found in literature. However, the refined precision allows now to exclude this electron-capture transition as a possible candidate for the determination of the neutrino mass.

Thus the PI-ICR technique appears very promising to tackle even more challenging cases such as ${ }^{134} \mathrm{Ce},{ }^{159} \mathrm{Dy}$ and ${ }^{175} \mathrm{Hf}$ [30, 31, the decay energy of which must be determined at a sub-100 eV level of precision.

Acknowledgements We thank the ISOLDE technical group and the ISOLDE Collaboration for their professional help. We acknowledge support by the Max Planck Society, the German Federal Ministry of Education and Research (BMBF) (05P12HGCI1, 05P12HGFNE, and 05P15ODCIA), the French IN2P3, the ExtreMe Matter Institute (EMMI) at GSI, and the European Union's Horizon 2020 research and innovation programme (654002). Jonas Karthein acknowledges the support by a Wolfgang Gentner Ph.D scholarship of the BMBF (05E12CHA). 


\section{References}

1. G. Bellini, L. Ludhova, G. Ranucci, and F. L. Villante. Neutrino Oscillations. Advances in High Energy Physics, 2014:1-28, jan 2014.

2. Carlo Giunti and Alexander Studenikin. Neutrino electromagnetic interactions: A window to new physics. Reviews of Modern Physics, 87(2):531-591, jun 2015.

3. O Ranitzsch, J.-P Porst, · S Kempf, · C Pies, · S Schäfer, · D Hengstler, and - A Fleischmann. Development of Metallic Magnetic Calorimeters for High Precision Measurements of Calorimetric 187 Re and 163 Ho Spectra. J Low Temp Phys, 167:1004-1014, 2012.

4. S Eliseev, K Blaum, M Block, S Chenmarev, H Dorrer, Ch E Düllmann, C Enss, P E Filianin, L Gastaldo, M Goncharov, U Köster, F Lautenschläger, Yu N Novikov, A Rischka, R X Schüssler, L Schweikhard, and A Türler. Direct Measurement of the Mass Difference of 163 Ho and 163 Dy Solves the Q-Value Puzzle for the Neutrino Mass Determination. 2015.

5. Y U Khazov, I Mitropolsky, and A Rodionov. Nuclear Data Sheets for A = 131. Nuclear Data Sheets, 107(11):2715-2930, 2006.

6. D Lunney and (on behalf of the ISOLTRAP Collaboration). Extending and refining the nuclear mass surface with ISOLTRAP. Journal of Physics G: Nuclear and Particle Physics, 44(6):064008, jun 2017.

7. S. Kreim, D. Atanasov, D. Beck, K. Blaum, Ch. Böhm, Ch. Borgmann, M. Breitenfeldt, T.E. Cocolios, D. Fink, S. George, A. Herlert, A. Kellerbauer, U. Köster, M. Kowalska, D. Lunney, V. Manea, E. Minaya Ramirez, S. Naimi, D. Neidherr, T. Nicol, R.E. Rossel, M. Rosenbusch, L. Schweikhard, J. Stanja, F. Wienholtz, R.N. Wolf, and K. Zuber. Recent exploits of the ISOLTRAP mass spectrometer. Nuclear Instruments and Methods in Physics Research Section B: Beam Interactions with Materials and Atoms, 317:492-500, dec 2013.

8. M. Mukherjee, D. Beck, K. Blaum, G. Bollen, J. Dilling, S. George, F. Herfurth, A. Herlert, A. Kellerbauer, H. J. Kluge, S. Schwarz, L. Schweikhard, and C. Yazidjian. ISOLTRAP: An on-line Penning trap for mass spectrometry on short-lived nuclides. The European Physical Journal A, 35(1):1-29, jan 2008.

9. María J G Borge and Klaus Blaum. Focus on Exotic Beams at ISOLDE: A Laboratory Portrait. Journal of Physics G: Nuclear and Particle Physics, 45(1):010301, jan 2018.

10. F Herfurth, J Dilling, A Kellerbauer, G Bollen, S Henry, H.-J Kluge, E Lamour, D Lunney, R.B Moore, C Scheidenberger, S Schwarz, G Sikler, and J Szerypo. A linear radiofrequency ion trap for accumulation, bunching, and emittance improvement of radioactive ion beams. Nuclear Instruments and Methods in Physics Research Section A: Accelerators, Spectrometers, Detectors and Associated Equipment, 469(2):254-275, aug 2001.

11. R.N. Wolf, F. Wienholtz, D. Atanasov, D. Beck, K. Blaum, Ch. Borgmann, F. Herfurth, M. Kowalska, S. Kreim, Yu. A. Litvinov, D. Lunney, V. Manea, D. Neidherr, M. Rosenbusch, L. Schweikhard, J. Stanja, and K. Zuber. ISOLTRAP's multi-reflection time-of-flight mass separator/spectrometer. International Journal of Mass Spectrometry, 349-350:123-133, sep 2013.

12. M Mougeot, D Atanasov, K Blaum, K Chrysalidis, T Day Goodacre, D Fedorov, V Fedosseev, S George, F Herfurth, J D Holt, D Lunney, V Manea, 
B Marsh, D Neidherr, M Rosenbusch, S Rothe, L Schweikhard, A Schwenk, C Seiffert, J Simonis, S R Stroberg, A Welker, F Wienholtz, R N Wolf, and K Zuber. Precision Mass Measurements of 58-63 Cr: Nuclear Collectivity Towards the $\mathrm{N}=40$ Island of Inversion. Physical Review Letters, 120:232501, 2018.

13. F. Wienholtz, D. Beck, K. Blaum, Ch. Borgmann, M. Breitenfeldt, R. B. Cakirli, S. George, F. Herfurth, J. D. Holt, M. Kowalska, S. Kreim, D. Lunney, V. Manea, J. Menéndez, D. Neidherr, M. Rosenbusch, L. Schweikhard, A. Schwenk, J. Simonis, J. Stanja, R. N. Wolf, and K. Zuber. Masses of exotic calcium isotopes pin down nuclear forces. Nature, 498(7454):346-349, jun 2013.

14. F. Wienholtz, S. Kreim, M. Rosenbusch, L. Schweikhard, and R.N. Wolf. Massselective ion ejection from multi-reflection time-of-flight devices via a pulsed in-trap lift. International Journal of Mass Spectrometry, 421:285-293, oct 2017.

15. G. Savard, St. Becker, G. Bollen, H.-J. Kluge, R.B. Moore, Th. Otto, L. Schweikhard, H. Stolzenberg, and U. Wiess. A new cooling technique for heavy ions in a Penning trap. Physics Letters A, 158(5):247-252, sep 1991.

16. M. König, G. Bollen, H.-J. Kluge, T. Otto, and J. Szerypo. Quadrupole excitation of stored ion motion at the true cyclotron frequency. International Journal of Mass Spectrometry and Ion Processes, 142(1-2):95-116, mar 1995.

17. S. George, S. Baruah, B. Blank, K. Blaum, M. Breitenfeldt, U. Hager, F. Herfurth, A. Herlert, A. Kellerbauer, H.-J. Kluge, M. Kretzschmar, D. Lunney, R. Savreux, S. Schwarz, L. Schweikhard, and C. Yazidjian. Ramsey Method of Separated Oscillatory Fields for High-Precision Penning Trap Mass Spectrometry. Physical Review Letters, 98(16):162501, apr 2007.

18. S. Eliseev, K. Blaum, M. Block, C. Droese, M. Goncharov, E. Minaya Ramirez, D. A. Nesterenko, Yu. N. Novikov, and L. Schweikhard. Phase-Imaging IonCyclotron-Resonance Measurements for Short-Lived Nuclides. Physical Review Letters, 110(8):082501, feb 2013.

19. S. Eliseev, K. Blaum, M. Block, A. Dörr, C. Droese, T. Eronen, M. Goncharov, M. Höcker, J. Ketter, E. Minaya Ramirez, D. A. Nesterenko, Yu. N. Novikov, and L. Schweikhard. A phase-imaging technique for cyclotron-frequency measurements. Applied Physics B, 114(1-2):107-128, jan 2014.

20. Jonas Karthein. Precision mass measurements using the Phase-Imaging IonCyclotron-Resonance detection technique - Master thesis - Ruprecht-KarlsUniversität Heidelberg, 2017.

21. I. Antcheva, M. Ballintijn, B. Bellenot, M. Biskup, R. Brun, N. Buncic, Ph. Canal, D. Casadei, O. Couet, V. Fine, L. Franco, G. Ganis, A. Gheata, D. Gonzalez Maline, M. Goto, J. Iwaszkiewicz, A. Kreshuk, D. Marcos Segura, R. Maunder, L. Moneta, A. Naumann, E. Offermann, V. Onuchin, S. Panacek, F. Rademakers, P. Russo, and M. Tadel. ROOT - A C++ framework for petabyte data storage, statistical analysis and visualization. Computer Physics Communications, 180(12):2499-2512, dec 2009.

22. D. Fink, J. Barea, D. Beck, K. Blaum, Ch. Böhm, Ch. Borgmann, M. Breitenfeldt, F. Herfurth, A. Herlert, J. Kotila, M. Kowalska, S. Kreim, D. Lunney, S. Naimi, M. Rosenbusch, S. Schwarz, L. Schweikhard, F. Šimkovic, J. Stanja, and K. Zuber. Q Value and Half-Lives for the Double- $\beta$-Decay Nuclide Pd 110. Physical Review Letters, 108(6):062502, feb 2012. 
23. Meng Wang, G. Audi, F. G. Kondev, W.J. Huang, S. Naimi, and Xing Xu. The AME2016 atomic mass evaluation (II). Tables, graphs and references. Chinese Physics C, 41(3):030003, mar 2017.

24. S. Sturm, F. Köhler, J. Zatorski, A. Wagner, Z. Harman, G. Werth, W. Quint, C. H. Keitel, and K. Blaum. High-precision measurement of the atomic mass of the electron. Nature, 506(7489):467-470, feb 2014.

25. A. Kellerbauer, K. Blaum, G. Bollen, F. Herfurth, H.-J. Kluge, M. Kuckein, E. Sauvan, C. Scheidenberger, and L. Schweikhard. From direct to absolute mass measurements: A study of the accuracy of ISOLTRAP. The European Physical Journal D - Atomic, Molecular and Optical Physics, 22(1):53-64, jan 2003.

26. A Welker, P Filianin, N A S Althubiti, D Atanasov, K Blaum, T E Cocolios, S Eliseev, F Herfurth, S Kreim, D Lunney, V Manea, D Neidherr, Yu Novikov, M Rosenbusch, L Schweikhard, F Wienholtz, R N Wolf, and K Zuber. Precision electron-capture energy in $202 \mathrm{~Pb}$ and its relevance for neutrino mass determination.

27. M. Cardona and L. Ley, editors. Photoemission in Solids I, volume 26 of Topics in Applied Physics. Springer Berlin Heidelberg, Berlin, Heidelberg, 1978.

28. NIST X-ray Photoelectron Spectroscopy (XPS) Database, Version 3.5.

29. D. A. Nesterenko, S. Eliseev, K. Blaum, M. Block, S. Chenmarev, A. Dörr, C. Droese, P. E. Filianin, M. Goncharov, E. Minaya Ramirez, Yu. N. Novikov, L. Schweikhard, and V. V. Simon. Direct determination of the atomic mass difference of Re 187 and Os 187 for neutrino physics and cosmochronology. Physical Review C, 90(4):042501, oct 2014.

30. S Eliseev, Numa Althubiti, P Ascher, D Atanasov, K Blaum, Ch Böhm, Ch Borgmann, M Breitenfeldt, R B Cakirli, T E Cocolios, T Eronen, P Filianin, S George, M Goncharov, F Herfurth, A Herlert, D Kisler, M Kowalska, S Kreim, Yu A Litvinov, D Lunney, V Manea, E Minaya Ramirez, S Naimi, D Neidherr, Yu Novikov, M Rosenbusch, A De Roubin, L Schweikhard, F Wienholtz, A Welker, R N Wolf, and K Zuber. Proposal to the ISOLDE and Neutron Time-of-Flight Committee: Search for $\beta$-transitions with the lowest decay energy for a determination of the neutrino mass. Technical report, CERN, 2014.

31. J Karthein, D Atanasov, K Blaum, S Eliseev, S George, F Herfurth, A Herlert, D Lunney, V Manea, M Mougeot, D Neidherr, Y Novikov, M Rosenbusch, L Schweikhard, F Wienholtz, A Welker, R Wolf, and K Zuber. Letter of Intent to the ISOLDE and Neutron Time-of-Flight Committee. Technical report, CERN, 2017. 